\title{
LOCAL VARIATIONS OF THE VELOCITY ELLIPSOID
}

\author{
M. MORENO \\ Dep. Fsica i Enginyeria Nuclear \\ Universitat Politècnica de Catalunya (Spain) \\ [manel@fen.upc.es] \\ J. TORRA \\ Dep. d'Astronomia i Meteorologia \\ Universitat de Barcelona (Spain) \\ AND \\ E. OBLAK \\ Observatoire de Besançon (France)
}

1. The data We have analyzed the distribution of residual velocities of nearby stars (within $200 \mathrm{pc}$ of the Sun) looking for space variations on the velocity ellipsoid. We used a sample of 1071 main sequence stars of spectral types B, A and F selected from the Hipparcos Input Catalogue [7] with $u v b y H_{\beta}$ photometric data. Ages have been calculated following [1]. Six subsamples with $8.07 \leq \log ($ age $) \leq 9.45$ have been considered.

2. Method and results The velocity distribution of stars in the solar neighborhood is described by a systematic part including the solar motion and galactic rotation, and a random part constituted by the peculiar velocities of the stars and the observational errors. The variances of peculiar velocities are expressed in terms of the velocity ellipsoid parameters that we assume to vary linearly with the position ([4], [6]). For each stellar sample, we simultaneously derive the solar motion, the velocity ellipsoid and its gradients in the galactic plane and in the z-direction. We use a new 3D extended version of the 2D algorithm developed by one of us [5].

The most remarkable results can be summarized as follows:

No dependence with age of the parameters of solar motion is found. We obtain the well known relations between the three semiaxes: $\sigma_{U}>\sigma_{V}>\sigma_{W}$, and the increase of their values when advancing toward older subsamples. The vertex deviation reaches its maximum values for samples number 2 : 
$\phi=20.9^{\circ} \pm 6.2^{\circ}$ and number $3: \phi=23.0^{\circ} \pm 5.7^{\circ}$, becoming null after twelve galactic revolutions (sample 6). The dependence of the vertex deviation on $\sigma_{U}$ for different age samples, agrees with [2], who suggested a possible connection between the vertex deviation and a local perturbation of mass density, which could be the Orion arm.

Despite of the values obtained for the gradients are small, some of them can be considered as significant and comparable to those founded by [5]. Due to the very large error bars on derived gradients, no significant ones can be obtained but for sample 2 (table 1) and, perhaps, sample 6. Sample 2 has the greatest number of significant gradients as in [5], this is the largest sample and is constituted by relatively young stars. The dispersion of the $U$ component decreases in the direction of the galactic center and increases in the direction of galactic rotation. The vertex deviation decreases in the direction of the galactic rotation and increases in the direction of the galactic center. The deformations and changes of orientation of the velocity ellipsoid are in good agreement with Oblak's results obtained for similar samples on the galactic plane. The gradients of the dispersion on the third component are all weak.

TABLE 1. Gradients of the velocity ellipsoid for sample 2 (321 stars, $8.37 \leq \log ($ age $) \leq 8.67)$ expressed in $\mathrm{km}^{2} / \mathrm{s}^{2} \cdot p c$

\begin{tabular}{ccccc}
\hline$\partial \mu_{200} / \partial \xi$ & $\partial \mu_{200} / \partial \eta$ & $\partial \mu_{020} / \partial \xi$ & $\partial \mu_{020} / \partial z$ & $\partial \mu_{110} / \partial z$ \\
\hline$-.83 \pm .41$ & $.62 \pm .34$ & $.23 \pm .23$ & $.43 \pm .17$ & $.27 \pm .27$ \\
\hline
\end{tabular}

Assuming the lines of constant vertex deviation as isoperturbation lines, [5] postulate the existence of a local structure oriented in the longitude direction $45^{\circ}-225^{\circ}$ as an acceptable cause of this behavior (the Sun located in the inner edge of the structure). This is in acceptable agreement with the direction of the Orion arm. A similar position is adopted by [3]. This work has been supported by CICYT ESP94-1311-E and DGICYT PB91-0857.

\section{References}

1. Asian, R. (1993), Degree in Physics, Universitat de Barcelona

2. Mayor, M. (1970), $A \& A$ 6, 60

3. Menge de Freitas, S. (1982), A\&A Suppl. Ser. 49, 687

4. Moreno, M., Torra, J. (1989), in Errors, bias and uncertainties in Astronomy C. Jaschek and F. Murtagh (eds.), Cambridge University Press, p. 377

5. Oblak, E. (1983), $A \& A$ 123, 238

6. Oblak, E., Moreno, M., Torra, J. (1993), in Workshop on Databases for Galactic Structure, Swarthmore (USA) (in press)

7. Turon, C. and 53 collaborators (1992), The Hipparcos Input Catalogue, ESA SP-1136 